\title{
Springback Analysis in Sheet Metal Forming Using Modified Ludwik Stress-Strain Relation
}

\author{
Sanjay Kumar Patel, Radha Krishna Lal, J. P. Dwivedi, and V. P. Singh \\ Department of Mechanical Engineering, Indian Institute of Technology, Banaras Hindu University, Varanasi 221005, India
}

Correspondence should be addressed to Radha Krishna Lal; radhakrishna773@gmail.com

Received 18 June 2013; Accepted 17 July 2013

Academic Editors: C. C. Huang and A. Z. Sahin

Copyright (C) 2013 Sanjay Kumar Patel et al. This is an open access article distributed under the Creative Commons Attribution License, which permits unrestricted use, distribution, and reproduction in any medium, provided the original work is properly cited.

\begin{abstract}
This paper deals with the springback analysis in sheet metal forming using modified Ludwik stress-strain relation. Using the deformation theory of plasticity, formulation of the problem and spring back ratio is derived using modified Ludwik stress strain relationship with Tresca and von Mises yielding criteraia. The results have been representing the effect of different value of $Y / E$ or $\sigma_{o} / E$ ratio, different values of Strain hardening index $(n)$, Poisson's ratio $(\nu)$, and thickness on spring back ratio $\left(R_{o} / R_{f}\right)$. The main aim of this paper is to study the effects of the thickness, $Y / E$ ratio, $n$ and Poisson's ratio in spring back ratio.
\end{abstract}

\section{Introduction}

In sheet metal working, sheets are deformed to cylindrical and helical shapes by plastic bending with the help of a punch and die set. When such bending is properly done, the inside contour of the section matches the surface contour of the die during the forming operation. However, after the release of the applied loads, the contour assumes a different shape than that of the die because of the release of elastic stresses in the metal. This elastic distortion is commonly called springback. Springback complicates tool design in that the die must be designed to compensate for it. Consequently, it is desirable to have a method of quantitatively predicting the magnitude of springback as a function of the properties of the material and geometry involved in the forming operation.

In bending, the springback is a measure of elastic recovery of radius on removal of the applied bending moment or load after the bending section is beyond elastic limit. While designing the die set, the springback factor should be taken into account to avoid mismatch while assembling formed different sections.

Initially, springback studies are with sheet bending operations. Sachs [1], Schroeder [2], Gardiner [3], Singh and Johnson [4], and others studied the springback considered bending of sheets of different shapes and depicted springback as a function of material thickness, length, and width of the sheets taken. Their studies were limited to V-and U-shaped dies for applying bending loads and they predicted the springback as a measure of change in the curvature distribution. Huth [5], Nadai [6], and Upadhyay [7] have all done a number of excellent work on the elasto-plastic torsion of bars with rectangular sections, but their work has been limited to monotonically increasing loads only. Dwivedi et al. $[8,9]$ analytically predicted the residual angle of twist and torque relation, and so forth, for bars of elastic strainhardening materials with narrow rectangular sections. This work, however, has the limitation that it is valid for thin rectangular strips only. Dwivedi et al. [10,11] dealt with the torsional springback of square-section bars of linear and nonlinear work-hardening materials. Dwivedi et al. [12] also dealt with the torsional springback of L-shaped section bars of nonlinear work-hardening materials.

An accurate analysis of springback has been made in the past on sheet bending and tube bending operations through experiment [13-18]. Torsional springback in thin tubes with nonlinear work hardening analysis was made by Choubey et al. [19-21].

In the following, approximation equations are derived in an attempt to provide a quantitative method with practical utility for predicting the springback behavior in bent section of sheet metal as a function of die radius, sheet thickness, and stress-strain characteristics of the material. 


\section{Basic Equations and Formulation}

In this paper, springback prediction approach using the modified Ludwik stress-strain relation was studied. The assumption of narrow beam of a wide sheet can significantly alter the magnitude of the predicted results in sheet metal bending because this ignorance of the transverse stresses are present during forming. In this paper, it is assumed that the bend section is a wide sheet of an elastic plastic strain hardening material in an effort to obtain more accurate expression for predicting the springback behavior. In this method, use of applied moment and curvature relation during the formation of a wide sheet of metal around a portion of cylindrical die was done to derive springback relationship. From the bending equation, we directly relate the bending moment and curvature during the forming as follows:

$$
\begin{gathered}
\frac{M}{I}=\frac{\sigma}{y}=\frac{E}{R}, \\
\frac{M}{E I}=\frac{1}{R}, \\
M=C * \frac{1}{R} .
\end{gathered}
$$

From this equation, the springback is related to the applied bending moment and curvature during the formation. The modified Ludwik stress-strain relation (Figure 2) is given by

$$
\sigma=\left[\begin{array}{cc}
E \varepsilon & \varepsilon \leq \frac{Y}{E} \\
Y\left(\frac{E \varepsilon}{Y}\right)^{n} & \varepsilon \geqslant \frac{Y}{E}
\end{array}\right],
$$

where $Y=\sigma_{o}, \sigma=E \varepsilon$ is the elastic part and $\sigma=\sigma_{o}\left(E \varepsilon / \sigma_{o}\right)^{n}$ is the plastic part, and the equation gives the total stress.

\section{Assumption for Derivation of Springback Behaviour}

(1) Pure bending of the sheet to cylindrical surface.

(2) The cross section dimensions of the sheet are such so that the width to thickness ratio is large.

(3) The stress-strain characteristics of material are the same in tension and compression.

(4) The natural surface is always in the centre of the sheet, and plane sections remain plane during bending.

(5) The cross section dimensions of the sheet do not change significantly in bending.

(6) The radius of bending is large compared to the thickness of the sheet so radial stresses are assumed to be negligible.

(7) The circumferential strains are sufficiently small so that the conventional strain and the true strain are approximately equivalent.

(8) The transverse strain is zero at any point in the sheet.

(9) The circumferential strain for any fiber does not vary along the bent section.

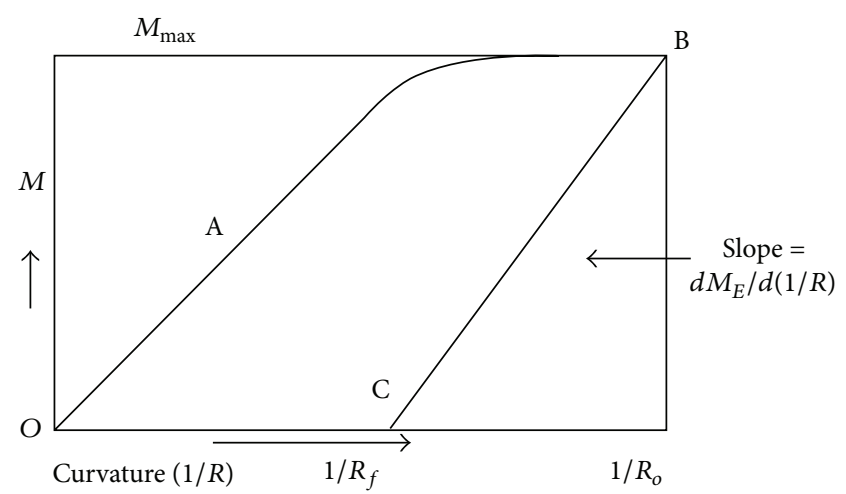

FIGURE 1: Schematic representation of curvature versus applied moment during bending.

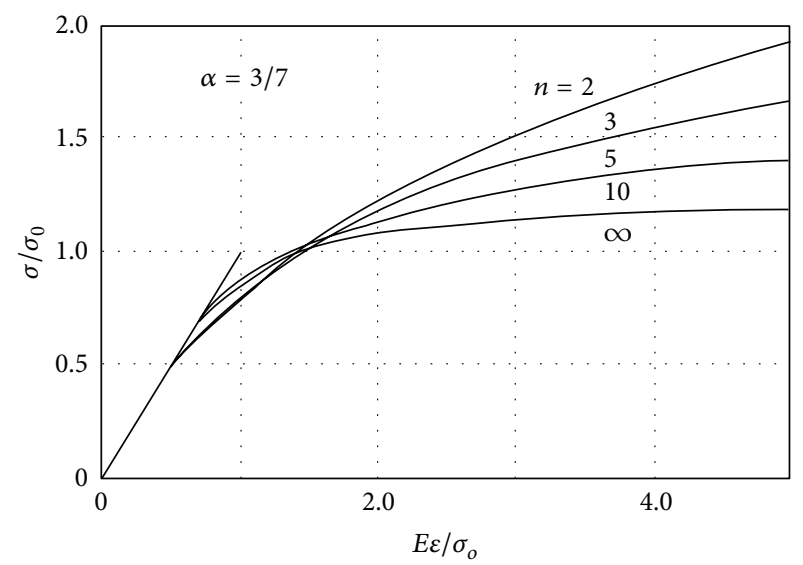

FIGURE 2: Empirical stress-strain curve for elastic plastic material in modified ludwik stress-strain relation.

\section{Derivation of Springback Equation for Tresca Yield Criteria}

Figure 1 shows the schematic plot of applied bending moment versus curvature during the formation of a wide sheet of metal around a portion of a cylindrical die at point $\mathrm{A}$, the material yields and plastic deformation continuous until the inside surface of the material conforms to the inside surface of the material conforms to die at point $B$ when the applied moment is released elastic springback occurs from $B$ to $C$. The change in curvature due to this elastic springback is $\left(1 / R_{o}\right)-\left(1 / R_{f}\right)$ From Figure 3,

$$
\frac{1}{R_{o}}-\frac{1}{R_{f}}=\frac{M_{\max }}{\partial M_{E} / \partial(1 / R)} .
$$

Figure 3 shows a section of bent sheet and the coordinate system adopted. Then calculation of maximum applied bending moment by balancing the external and internal moments is as follows:

$$
M_{\max }=\int_{-t / 2}^{+t / 2} \sigma_{x} y d y=2 \int_{0}^{t / 2} \sigma_{x} y d y .
$$




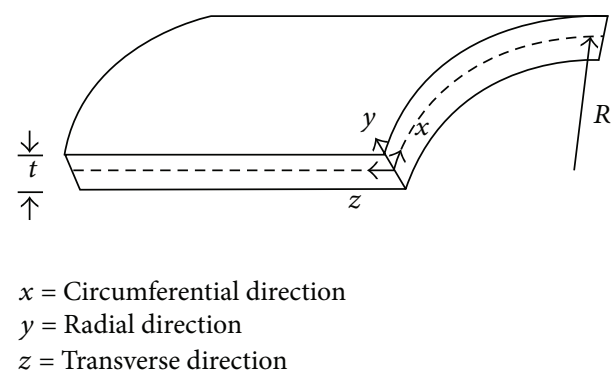

FIGURE 3: Direction of principal stresses and curvature during bending.

Before proceeding, it is desirable to attempt to characterize the stress-strain behaviour of material in a simple tension. From the modified Ludwik stress-strain relation, we get

$$
\sigma=\left[\begin{array}{cc}
E \varepsilon & \varepsilon \leq \frac{Y}{E} \\
Y\left(\frac{E \varepsilon}{Y}\right)^{n} & \varepsilon \geqslant \frac{Y}{E}
\end{array}\right] .
$$

This is the equation for springback for Modified Ludwik stress-strain relation.

In elastic region,

$$
\sigma=E \varepsilon
$$

In plastic region, where $Y=\sigma_{o}$,

$$
\sigma=\sigma_{o}\left(\frac{E}{\sigma_{o}}\right)^{n} \varepsilon^{n} .
$$

Let

$$
\sigma_{o}\left(\frac{E}{\sigma_{o}}\right)^{n}=K
$$

Then

$$
\sigma=K \varepsilon^{n}
$$

At yield point, the stress in elastic and plastic region is equal; so we can write

$$
\begin{gathered}
E \varepsilon_{o}=K \varepsilon_{o}^{n}, \\
\varepsilon_{o}=\left(\frac{K}{E}\right)^{1 /(1-n)} .
\end{gathered}
$$

From (10), stress in yield point is

$$
\sigma_{o}=K \varepsilon_{o}^{n}=K\left(\frac{K}{E}\right)^{n /(1-n)} .
$$

It will be assumed for stresses less than $\sigma_{o}$ that the stress-strain behaviour of the material in a simple tension test is elastic (6) and that yielding occurs at $\sigma_{o}$ and (7) is applicable for stresses greater than $\sigma_{o}$.
For combined states of stress, the relationship between principal stresses and strain for the elastic.

By Hooke's law,

$$
\begin{aligned}
& \varepsilon_{x}=\frac{1}{E}\left(\sigma_{x}-\nu\left(\sigma_{y}+\sigma_{z}\right)\right), \\
& \varepsilon_{y}=\frac{1}{E}\left(\sigma_{y}-\nu\left(\sigma_{x}+\sigma_{z}\right)\right), \\
& \varepsilon_{z}=\frac{1}{E}\left(\sigma_{z}-\nu\left(\sigma_{y}+\sigma_{x}\right)\right) .
\end{aligned}
$$

From assumptions that the radius of bending is large compared to thickness of the sheet so radial stresses can assume to be negligible, and the transverse strain is zero at any point in the sheet,

$$
\sigma_{y}=\varepsilon_{z}=\delta_{z}=0 .
$$

And the circumferential strain for any fiber does not vary along the bent section.

So,

$$
\varepsilon_{x}=\delta_{x}
$$

Then, from putting the value of (12) in (13),

$$
\begin{gathered}
\varepsilon_{z}=\frac{1}{E}\left(\sigma_{z}-\nu\left(\sigma_{y}+\sigma_{x}\right)\right), \\
0=\frac{1}{E}\left(\sigma_{z}-\nu\left(\sigma_{y}+\sigma_{x}\right)\right), \\
\frac{1}{E}\left(\sigma_{z}-\nu\left(\sigma_{x}\right)\right)=0, \\
\sigma_{z}=\nu \sigma_{x} .
\end{gathered}
$$

From maximum shear stress theory of failure (Tresca yield criteria),

$$
\begin{gathered}
\frac{\sigma_{o}}{2}=\frac{\sigma_{x}-\sigma_{z}}{2}, \\
\sigma_{o}=\sigma_{x}-\nu \sigma_{x}, \\
\sigma_{o}=\sigma_{x}(1-\nu) .
\end{gathered}
$$

Substituting (16) in (11),

$$
\begin{gathered}
\sigma_{o}=\sigma_{x}(1-v)=K\left(\frac{K}{E}\right)^{n /(1-n)}, \\
\sigma_{x}=\frac{K(K / E)^{1 /(1-n)}}{(1-v)} .
\end{gathered}
$$

Then,

$$
\begin{gathered}
\varepsilon_{x}=\frac{1}{E}\left(\sigma_{x}-\nu\left(\sigma_{y}+\sigma_{z}\right)\right), \\
\varepsilon_{x}=\frac{1}{E}\left(\sigma_{x}-v^{2} \sigma_{x}\right), \\
\varepsilon_{x}=\left(\frac{1}{E}\right) \sigma_{x}\left(1-v^{2}\right) .
\end{gathered}
$$


So in yield point, the circumferential strain is

$$
\varepsilon_{x o}=\left(\frac{1}{E}\right) \sigma_{o}\left(1-v^{2}\right) .
$$

Putting yield point stress value from (11), we get

$$
\begin{gathered}
\varepsilon_{x o}=\frac{K(K / E)^{n /(1-n)}}{E(1-\nu)}\left(1-\nu^{2}\right), \\
\varepsilon_{x o}=\left(\frac{K}{E}\right)^{1 /(1-n)}(1+\nu) .
\end{gathered}
$$

This is the approximate value of circumferential strain at elastic plastic interface. Then in elastic region, the circumferential stress is

$$
\sigma_{x}=\frac{E}{\left(1-v^{2}\right)} \varepsilon_{x} .
$$

Here, $\varepsilon_{x}=y / R_{o}$.

Then,

$$
\sigma_{x}=\frac{E}{\left(1-v^{2}\right)}\left(\frac{y}{R_{o}}\right),
$$

for $0 \leq \varepsilon_{x} \leq(K / E)^{1 /(1-n)}(1+\nu)$.

From the deformation theory of plasticity,

$$
\begin{aligned}
\delta_{x}= & \frac{1}{K^{1 / n}}\left(\sigma_{x}^{2}+\sigma_{y}^{2}+\sigma_{z}^{2}-\sigma_{x} \sigma_{y}-\sigma_{y} \sigma_{z}-\sigma_{z} \sigma_{x}\right)^{(1-n) / 2 n} \\
& \times\left(\sigma_{x}-\frac{\sigma_{y}}{2}-\frac{\sigma_{z}}{2}\right), \\
\delta_{y}= & \frac{1}{K^{1 / n}}\left(\sigma_{x}^{2}+\sigma_{y}^{2}+\sigma_{z}^{2}-\sigma_{x} \sigma_{y}-\sigma_{y} \sigma_{z}-\sigma_{z} \sigma_{x}\right)^{(1-n) / 2 n} \\
& \times\left(\sigma_{y}-\frac{\sigma_{x}}{2}-\frac{\sigma_{z}}{2}\right) \\
\delta_{z}= & \frac{1}{K^{1 / n}}\left(\sigma_{x}^{2}+\sigma_{y}^{2}+\sigma_{z}^{2}-\sigma_{x} \sigma_{y}-\sigma_{y} \sigma_{z}-\sigma_{z} \sigma_{x}\right)^{(1-n) / 2 n} \\
& \times\left(\sigma_{z}-\frac{\sigma_{x}}{2}-\frac{\sigma_{y}}{2}\right) .
\end{aligned}
$$

From assumptions that the radius of bending is large compared to thickness of the sheet so radial stresses can assume to be negligible and the transverse strain is zero at any point in the sheet, $\sigma_{y}=\varepsilon_{z}=\delta_{z}=0$.

And the circumferential strain for any fiber does not vary along the bent section. So, $\varepsilon_{x}=\delta_{x}$.

Put this value in (23). We get

$$
\begin{aligned}
\delta_{z}= & \frac{1}{K^{1 / n}}\left(\sigma_{x}^{2}+\sigma_{y}^{2}+\sigma_{z}^{2}-\sigma_{x} \sigma_{y}-\sigma_{y} \sigma_{z}-\sigma_{z} \sigma_{x}\right)^{(1-n) / 2 n} \\
& \times\left(\sigma_{z}-\frac{\sigma_{x}}{2}-\frac{\sigma_{y}}{2}\right) \\
0 & =\frac{1}{K^{1 / n}}\left(\sigma_{x}^{2}+\sigma_{z}^{2}-\sigma_{z} \sigma_{x}\right)^{(1-n) / 2 n}\left(\sigma_{z}-\frac{\sigma_{x}}{2}\right) .
\end{aligned}
$$

So,

$$
\begin{gathered}
0=\left(\sigma_{z}-\frac{\sigma_{x}}{2}\right), \\
\sigma_{z}=\frac{\sigma_{x}}{2} .
\end{gathered}
$$

Putting the value of $\sigma_{z}$ into (23), we found that

$$
\begin{gathered}
\delta_{x}=\frac{1}{K^{1 / n}}\left(\sigma_{x}^{2}+\sigma_{y}^{2}+\sigma_{z}^{2}-\sigma_{x} \sigma_{y}-\sigma_{y} \sigma_{z}-\sigma_{z} \sigma_{x}\right)^{(1-n) / 2 n} \\
\times\left(\sigma_{x}-\frac{\sigma_{y}}{2}-\frac{\sigma_{z}}{2}\right) \\
\delta_{x}=\frac{1}{K^{1 / n}}\left(\sigma_{x}^{2}+\frac{\sigma_{x}^{2}}{4}-\frac{\sigma_{x}^{2}}{2}\right)^{(1-n) / 2 n}\left(\sigma_{x}-\frac{\sigma_{x}}{4}\right), \\
\delta_{x}=\frac{1}{K^{1 / n}}\left(\frac{3 \sigma_{x}^{2}}{4}\right)^{(1-n) / 2 n}\left(\frac{3 \sigma_{x}}{4}\right), \\
\delta_{x}=\frac{1}{K^{1 / n}}\left(\frac{3}{4}\right)^{(1+n) / 2 n} \sigma_{x}^{1 / n} \\
\sigma_{x}=\frac{K}{(3 / 4)^{(1+n) / 2}} \delta_{x}^{n} .
\end{gathered}
$$

From initial assumption that the circumferential strain are sufficiently small so that the conventional strain and true strain are approximately equivalent,

$$
\varepsilon_{x}=\frac{y}{R_{o}}=\delta_{x}
$$

Putting the value of $\delta_{x}$ into (26), we get

$$
\sigma_{x}=\frac{K}{(3 / 4)^{(1+n) / 2}}\left(\frac{y}{R_{o}}\right)^{n},
$$

where $\sigma_{x}$ is valid for plastic region; that is,

$$
\left(\frac{K}{E}\right)^{1 /(1-n)}(1+\nu) \leq \varepsilon_{x} \leq \frac{t}{2}
$$

From (4), we found maximum bending moment is

$$
M_{\max }=2\left[\int_{0}^{t / 2} \sigma_{x} y d y\right],
$$

where we can split the limit into elastic or plastic part, from 0 to $R_{\text {oex }}$ elastic region and $R_{\text {oex }}$ to $(t / 2)$ plastic region, where $R_{\text {oex }}$ is calculated by

$$
R_{o e x}=R_{o}\left(\frac{K}{E}\right)^{1 /(1-n)}(1+v)
$$


Now,

$$
\begin{aligned}
M_{\max }= & 2\left[\int_{0}^{R_{\text {oex }}} \sigma_{x \text { (elastic) }} y d y+\int_{R_{\text {oex }}}^{t / 2} \sigma_{x \text { (plastic) }} y d y\right] \\
= & 2\left[\int_{0}^{R_{\text {oex }}}\left(\frac{E}{1-v^{2}}\right) y^{2} d y\right. \\
& \left.+\int_{R_{\text {oex }}}^{t / 2} \frac{K}{(3 / 4)^{(1+n) / 2}}\left(\frac{y}{R_{o}}\right)^{n} y d y\right] \\
= & 2\left[\int_{0}^{R_{\text {oex }}}\left(\frac{E}{1-v^{2}}\right) y^{2} d y\right. \\
& \left.+\int_{R_{\text {oex }}}^{t / 2} \frac{K}{(3 / 4)^{(1+n) / 2}}\left(\frac{1}{R_{o}}\right)^{n} y^{n+1} d y\right] \\
= & 2\left(\frac{1}{R_{o}}\right)\left[\left(\frac{E}{1-v^{2}}\right) \frac{y^{3}}{3}\right]_{0}^{R_{\text {oex }}} \\
& +2\left[\frac{K}{(3 / 4)^{(1+n) / 2}}\left(\frac{1}{R_{o}}\right)^{n} \frac{y^{n+2}}{n+2}\right]_{R_{\text {oex }}}^{t / 2}
\end{aligned}
$$

Put the value of $R_{\text {oex }}$ in above equation.

Then

$$
\begin{gathered}
=2\left[\frac{E R_{o}^{2}(K / E)^{3 /(1-n)}}{3(1-v)}(1+\nu)^{2}\right] \\
+2\left[\frac{K(t / 2)^{n+2}}{(3 / 4)^{(1+n) / 2} R_{o}^{n}(n+2)}\right. \\
\left.-\frac{K R_{o}^{2}(K / E)^{(n+2) /(1-n)}(1+\nu)^{n+2}}{(3 / 4)^{(1+n) / 2}(n+2)}\right] .
\end{gathered}
$$

From elementary plate theory [17] applied moment per unit width during elastic unloading,

$$
\begin{gathered}
\frac{M_{E}}{I}=\frac{\sigma_{x}}{y}, \\
M_{E}=\frac{2 \cdot E \cdot(t / 2)^{3}}{3\left(1-v^{2}\right) \cdot R} .
\end{gathered}
$$

The slope of elastic recovery in elastic unloading is

$$
\frac{\partial M_{E}}{\partial(1 / R)}=\frac{2 \cdot E \cdot(t / 2)^{3}}{3\left(1-v^{2}\right)} .
$$

Putting this into (3), we get

$$
\begin{aligned}
& \frac{1}{R_{o}}-\frac{1}{R_{f}}=\frac{M_{\max }}{\partial M_{E} / \partial(1 / R)} \frac{R_{o}}{R_{f}}=1-R_{o}\left(\frac{M_{\max }}{\partial M_{E} / \partial(1 / R)}\right), \\
& \frac{R_{o}}{R_{f}}=1-R_{o}\left\{\left(\frac{2 R_{o}}{t}\right)^{3}\left(\frac{K}{E}\right)^{3 /(1-n)}(1+v)^{3}\right. \\
& +\frac{3 K\left(1-v^{2}\right)\left(2 R_{o} / t\right)^{1-n}}{E(3 / 4)^{(1+n) / 2}(n+2)} \\
& \left.-\frac{3\left(\left(2 R_{o}\right) / t\right)^{3}(K / E)^{3 /(1-n)}(1+\nu)^{n+3}(1-\nu)}{(3 / 4)^{(1+n) / 2}(n+2)}\right\} \text {, } \\
& \frac{R_{o}}{R_{f}}=1-\frac{3 K\left(1-v^{2}\right)}{E(3 / 4)^{(1+n) / 2}(n+2)}\left(\frac{2 R_{o}}{t}\right)^{1-n} \\
& +\left[\left(\frac{2 R_{o}}{t}\right)\left(\frac{K}{E}\right)^{1 /(1-n)}(1+v)\right]^{3} \\
& \times\left[\frac{3(1+v)^{n}(1-v)}{(3 / 4)^{(1+n) / 2}(n+2)}-1\right] \text {, } \\
& \frac{R_{o}}{R_{f}}=1-\frac{3\left(1-v^{2}\right)}{(3 / 4)^{(1+n) / 2}(n+2)}\left(\frac{E}{\sigma_{o}}\right)^{n-1}\left(\frac{2 R_{o}}{t}\right)^{1-n} \\
& +\left[\left(\frac{2 R_{o}}{t}\right)\left(\frac{\sigma_{o}}{E}\right)^{1}(1+v)\right]^{3} \\
& \times\left[\frac{3(1+v)^{n}(1-v)}{(3 / 4)^{(1+n) / 2}(n+2)}-1\right] \text {. }
\end{aligned}
$$

This is the equation for springback ratio for modified Ludwik stress-strain relationship using maximum shear stress theory.

\section{Derivation of Springback Equation for Von Mises Yielding}

From maximum shear stress theory of failure (von Mises yielding criteria),

$$
\begin{gathered}
\sigma_{o}^{2}=\frac{1}{2}\left\{\left(\sigma_{x}-\sigma_{y}\right)^{2}+\left(\sigma_{y}-\sigma_{z}\right)^{2}+\left(\sigma_{z}-\sigma_{x}\right)^{2}\right\} \\
\sigma_{o}^{2}=\frac{1}{2}\left\{\sigma_{x}^{2}+\sigma_{z}^{2}+\sigma_{z}^{2}-2 \sigma_{z} \sigma_{x}+\sigma_{x}^{2}\right\} \\
\sigma_{o}=\sigma_{x}\left(1+\nu^{2}-\nu\right)^{1 / 2}
\end{gathered}
$$

by using (13) and (14). 
Substituting (15) in (11), at yield point,

$$
\begin{gathered}
\sigma_{o}=K\left(\frac{K}{E}\right)^{n /(1-n)}=\sigma_{o x}\left(1+v^{2}-v\right)^{1 / 2}, \\
\sigma_{o x}\left(1+\nu^{2}-v\right)^{1 / 2}=K\left(\frac{K}{E}\right)^{n /(1-n)}, \\
\sigma_{x o}=\frac{K(K / E)^{n /(1-n)}}{\left(1-\nu+\nu^{2}\right)^{1 / 2}} .
\end{gathered}
$$

Now,

$$
\begin{gathered}
\varepsilon_{x}=\frac{1}{E}\left(\sigma_{x}-v\left(\sigma_{y}+\sigma_{z}\right)\right), \\
\varepsilon_{x}=\frac{1}{E}\left(\sigma_{x}-\nu^{2} \sigma_{x}\right), \\
\varepsilon_{x}=\frac{1}{E} \sigma_{x}\left(1-\nu^{2}\right) .
\end{gathered}
$$

So, in yield point, the circumferential strain is

$$
\varepsilon_{x o}=\frac{1}{E} \sigma_{o x}\left(1-v^{2}\right) \text {. }
$$

Putting yield point stress value from (38) in (40), we get

$$
\begin{gathered}
\varepsilon_{x o}=\frac{K(K / E)^{n /(1-n)}}{E\left(1-\vartheta+\vartheta^{2}\right)^{1 / 2}}\left(1-\nu^{2}\right), \\
\varepsilon_{x o}=\left(\frac{K}{E}\right)^{1 /(1-n)} \frac{\left(1-v^{2}\right)}{\left(1-v+v^{2}\right)^{1 / 2}} .
\end{gathered}
$$

This is the approximate value of circumferential strain at elastic plastic interface.

Then in elastic region, the circumferential stress is

$$
\sigma_{x}=\frac{E}{\left(1-v^{2}\right)} \varepsilon_{x} .
$$

Here, $\varepsilon_{x}=y / R_{o}$.

Putting value of circumferential strain, we get

$$
\sigma_{x}=\frac{E}{\left(1-v^{2}\right)}\left(\frac{y}{R_{o}}\right),
$$

for $0 \leq \varepsilon_{x} \leq(K / E)^{1 /(1-n)}\left(\left(1-v^{2}\right) /\left(1-v+v^{2}\right)^{1 / 2}\right)$.

From the deformation theory of plasticity [16],

$$
\begin{aligned}
\delta_{x}= & \frac{1}{K^{1 / n}}\left(\sigma_{x}^{2}+\sigma_{y}^{2}+\sigma_{z}^{2}-\sigma_{x} \sigma_{y}-\sigma_{y} \sigma_{z}-\sigma_{z} \sigma_{x}\right)^{(1-n) / 2 n} \\
& \times\left(\sigma_{x}-\frac{\sigma_{y}}{2}-\frac{\sigma_{z}}{2}\right), \\
\delta_{y}= & \frac{1}{K^{1 / n}}\left(\sigma_{x}^{2}+\sigma_{y}^{2}+\sigma_{z}^{2}-\sigma_{x} \sigma_{y}-\sigma_{y} \sigma_{z}-\sigma_{z} \sigma_{x}\right)^{(1-n) / 2 n} \\
& \times\left(\sigma_{y}-\frac{\sigma_{x}}{2}-\frac{\sigma_{z}}{2}\right) \\
\delta_{z}= & \frac{1}{K^{1 / n}}\left(\sigma_{x}^{2}+\sigma_{y}^{2}+\sigma_{z}^{2}-\sigma_{x} \sigma_{y}-\sigma_{y} \sigma_{z}-\sigma_{z} \sigma_{x}\right)^{(1-n) / 2 n} \\
& \times\left(\sigma_{z}-\frac{\sigma_{x}}{2}-\frac{\sigma_{y}}{2}\right) .
\end{aligned}
$$

From assumptions that the radius of bending is large compared to thickness of the sheet so radial stresses can assume to be negligible and the transverse strain is zero at any point in the sheet,

$$
\sigma_{y}=\varepsilon_{z}=\delta_{z}=0 \text {. }
$$

And the circumferential strain for any fiber does not vary along the bent section.

So,

$$
\varepsilon_{x}=\delta_{x} .
$$

Put these values in (44). We get

$$
\begin{aligned}
\delta_{z}= & \frac{1}{K^{1 / n}}\left(\sigma_{x}^{2}+\sigma_{y}^{2}+\sigma_{z}^{2}-\sigma_{x} \sigma_{y}-\sigma_{y} \sigma_{z}-\sigma_{z} \sigma_{x}\right)^{(1-n) / 2 n} \\
& \times\left(\sigma_{z}-\frac{\sigma_{x}}{2}-\frac{\sigma_{y}}{2}\right), \\
0 & =\frac{1}{K^{1 / n}}\left(\sigma_{x}^{2}+\sigma_{z}^{2}-\sigma_{z} \sigma_{x}\right)^{(1-n) / 2 n}\left(\sigma_{z}-\frac{\sigma_{x}}{2}\right) .
\end{aligned}
$$

So,

$$
\begin{gathered}
0=\left(\sigma_{z}-\frac{\sigma_{x}}{2}\right), \\
\sigma_{z}=\frac{\sigma_{x}}{2} .
\end{gathered}
$$

Putting the value of $\sigma_{z}$ into (44), we get

$$
\begin{gathered}
\delta_{x}=\frac{1}{K^{1 / n}}\left(\sigma_{x}^{2}+\sigma_{y}^{2}+\sigma_{z}^{2}-\sigma_{x} \sigma_{y}-\sigma_{y} \sigma_{z}-\sigma_{z} \sigma_{x}\right)^{(1-n) / 2 n} \\
\times\left(\sigma_{x}-\frac{\sigma_{y}}{2}-\frac{\sigma_{z}}{2}\right), \\
\delta_{x}=\frac{1}{K^{1 / n}}\left(\sigma_{x}^{2}+\frac{\sigma_{x}^{2}}{4}-\frac{\sigma_{x}^{2}}{2}\right)^{(1-n) / 2 n}\left(\sigma_{x}-\frac{\sigma_{x}}{4}\right), \\
\delta_{x}=\frac{1}{K^{1 / n}}\left(\frac{3 \sigma_{x}^{2}}{4}\right)^{(1-n) / 2 n}\left(\frac{3 \sigma_{x}}{4}\right), \\
\delta_{x}=\frac{1}{K^{1 / \lambda}}\left(\frac{3}{4}\right)^{(1+n) / 2 n} \sigma_{x}^{1 / n}, \\
\sigma_{x}=\frac{K}{(3 / 4)^{(1+n) / n}} \delta_{x}^{n} .
\end{gathered}
$$

From initial assumption that the circumferential strain are sufficiently small so that the conventional strain and true strain are approximately equivalent,

$$
\varepsilon_{x}=\frac{y}{R_{o}}=\delta_{x} .
$$

Putting the value of $\delta_{x}$ into (49), we get

$$
\sigma_{x}=\frac{K}{(3 / 4)^{(1+n) / n}}\left(\frac{y}{R_{o}}\right)^{n},
$$


where $\sigma_{x}$ is valid for plastic region; that is,

$$
\text { for }\left(\frac{K}{E}\right)^{1 /(1-n)} \frac{\left(1-v^{2}\right)}{\left(1-v+v^{2}\right)} \leq \varepsilon_{x} \leq \frac{t}{2} \text {. }
$$

From (4), maximum bending moment is

$$
M_{\max }=2\left[\int_{0}^{t / 2} \sigma_{x} y d y\right] .
$$

We can split the limit into elastic or plastic part from 0 to $t / 2$ to 0 to $R_{\text {oex }}$ elastic region and $R_{\text {oex }}$ to $t / 2$ plastic region where $R_{\text {oex }}$ is calculated by

$$
\begin{gathered}
R_{\text {oex }}=\left(\frac{K}{E}\right)^{1 /(1-n)} R_{o} \frac{\left(1-v^{2}\right)}{\left(1-v+v^{2}\right)^{1 / 2}}, \\
M_{\max }=2\left[\int_{0}^{R_{\text {oex }}} \sigma_{x \text { (elastic) }} y d y+\int_{R_{\text {oex }}}^{t / 2} \sigma_{x \text { (plastic) }} y d y\right] \\
=2\left[\int_{0}^{R_{\text {oex }}} \frac{E}{1-v^{2}} y^{2}\left(\frac{1}{R_{o}}\right) d y\right. \\
=2\left[\int_{0}^{R_{o e x}}\left(\frac{E}{1-v^{2}}\right) y^{2}\left(\frac{1}{R_{o}}\right) d y\right. \\
\left.+\int_{R_{o e x}}^{t / 2} \frac{K}{(3 / 4)^{(1+n) / 2}}\left(\frac{1}{R_{o}}\right)^{n} y^{n+1} d y\right] \\
+2\left(\frac{1}{R_{o}}\right)\left[\left(\frac{E}{1-v_{o}^{2}}\right) \frac{y^{3}}{3}\right]_{0}^{R_{o e x}} \\
+2\left[\frac{K}{(3 / 4)^{(1+n) / 2}}\left(\frac{1}{R_{o}}\right)^{n} \frac{y^{n+2}}{n+2}\right]_{R_{o e x}}^{t / 2}
\end{gathered}
$$

Putting the value of $R_{\text {oex }}$, we get

$$
R_{o e x}=\left(\frac{K}{E}\right)^{1 /(1-n)} R_{o} \frac{\left(1-v^{2}\right)}{\left(1-\nu+v^{2}\right)^{1 / 2}}
$$

Then

$$
\begin{aligned}
M_{\max }= & 2\left[\frac{E R_{o}^{3}(K / E)^{3 /(1-n)}\left(\left(1-v^{2}\right)^{3} /\left(1-v+v^{2}\right)^{3 / 2}\right)}{3\left(1-v^{2}\right) R_{o}}\right] \\
+ & 2\left[\frac{K(t / 2)^{n+2}}{(3 / 4)^{(1+n) / 2} R_{o}^{n}(n+2)}\right. \\
& \left.-\frac{K R_{o}^{(n+2)}(K / E)^{(n+2) /(1-n)}\left(1-v^{2}\right)^{n+2}}{(3 / 4)^{(1+n) / 2} R_{o}^{n}(n+2)\left(1-v+v^{2}\right)^{(n+2) / 2}}\right] .
\end{aligned}
$$

From elementary plate theory [17] applied moment per unit width during elastic unloading,

$$
M_{E}=\frac{2 \cdot E \cdot(t / 2)^{3}}{3\left(1-v^{2}\right) \cdot R} .
$$

Now, the slope of elastic recovery in elastic unloading is

$$
\frac{\partial M_{E}}{\partial(1 / R)}=\frac{2 \cdot E \cdot(t / 2)^{3}}{3\left(1-v^{2}\right)} .
$$

Putting this into (3), we get

$$
\begin{gathered}
\frac{1}{R_{0}}-\frac{1}{R_{f}}=\frac{M_{\max }}{\partial M_{E} / \partial(1 / R)}, \\
\frac{R_{o}}{R_{f}}=1-R_{o} \frac{M_{\max }}{\partial M_{E} / \partial(1 / R)}, \\
\frac{R_{o}}{R_{f}=1-} \frac{3 K\left(1-v^{2}\right)}{E(n+2)(3 / 4)^{(n+1) / 2}}\left(\frac{2 R_{o}}{t}\right)^{1-n} \\
\left.+\left[\frac{2 R_{o}}{t}\right)\left(\frac{K}{E}\right)^{1 /(1-n)}\right]^{3} \\
\times\left[\frac{3\left(1-v^{2}\right)^{n+3}}{(3 / 4)^{(n+1) / 2}(n+2)\left(1-v+v^{2}\right)^{(n+2) / 2}}\right. \\
{\left[\frac{\left(1-v^{2}\right)^{3}}{\left(1-v+v^{2}\right)^{3 / 2}}\right],}
\end{gathered}
$$

$$
\begin{aligned}
\frac{R_{o}}{R_{f}}= & 1-\frac{3\left(1-v^{2}\right)}{(n+2)(3 / 4)^{(n+1) / 2}}\left(\frac{\sigma_{o}}{E}\right)^{1-n}\left(\frac{2 R_{o}}{t}\right)^{1-n} \\
+ & {\left[\left(\frac{2 R_{o}}{t}\right)\left(\frac{\sigma_{o}}{E}\right)\right]^{3} } \\
\times & {\left[\frac{(3 / 4)^{(n+1) / 2}(n+2)\left(1-v+v^{2}\right)^{(n+2) / 2}}{\left(1-v^{2}\right)^{3}}\right] . } \\
& -\frac{\left(1-v+v^{2}\right)^{3 / 2}}{(1-3} .
\end{aligned}
$$

This is the equation for springback ratio for modified Ludwik stress-strain relationship using von Mises yield criteria. 


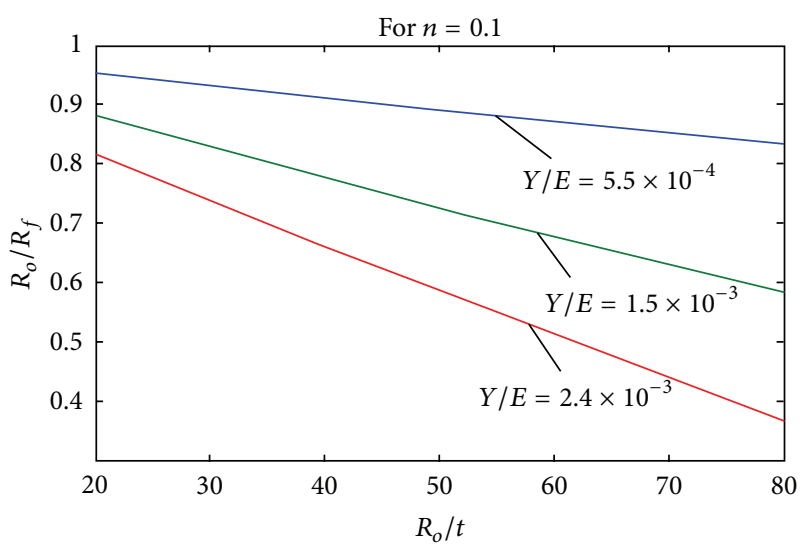

FIGURE 4: Springback ratio with $R_{o} / t$ with different values of $\sigma_{o} / E$ or $Y / E$ at $n=0.1, v=0.33$.

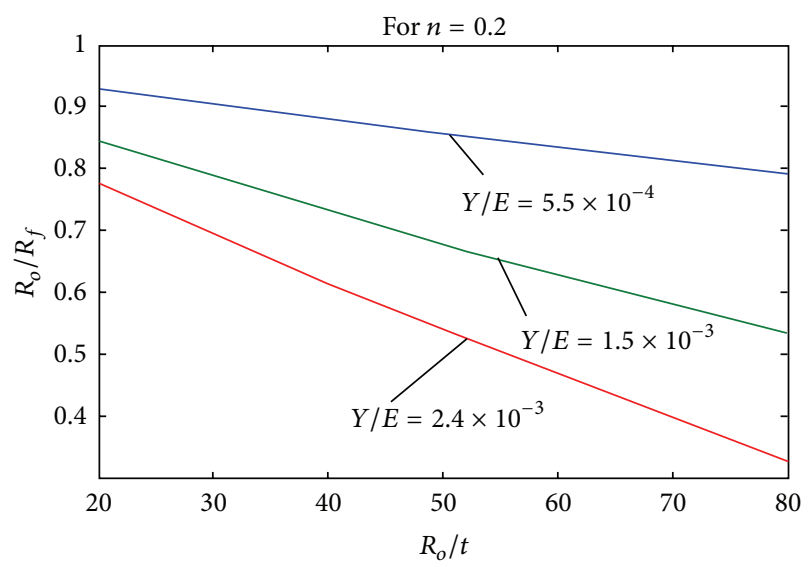

FIGURE 5: Springback ratio with $R_{o} / t$ with different values of $\sigma_{o} / E$ or $Y / E$ at $n=0.2, v=0.33$.

\section{Results and Discussion}

For the sheet metal bending, the springback is calculated from derived equation (using modified Ludwik stress-strain relationship with Tresca and von Mises yield criteria).

$$
\begin{aligned}
\frac{R_{o}}{R_{f}}=1 & -\frac{3\left(1-v^{2}\right)}{(3 / 4)^{(1+n) / 2}(n+2)}\left(\frac{E}{\sigma_{o}}\right)^{n-1}\left(\frac{2 R_{o}}{t}\right)^{1-n} \\
+ & {\left[\left(\frac{2 R_{o}}{t}\right)\left(\frac{\sigma_{o}}{E}\right)^{1}(1+v)\right]^{3} } \\
\times & {\left[\frac{3(1+v)^{n}(1-v)}{(3 / 4)^{(1+n) / 2}(n+2)}-1\right], } \\
\frac{R_{o}}{R_{f}}=1 & -\frac{3\left(1-v^{2}\right)}{(n+2)(3 / 4)^{(n+1) / 2}}\left(\frac{\sigma_{o}}{E}\right)^{1-n}\left(\frac{2 R_{o}}{t}\right)^{1-n} \\
+ & {\left[\left(\frac{2 R_{o}}{t}\right)\left(\frac{\sigma_{o}}{E}\right)\right]^{3} }
\end{aligned}
$$

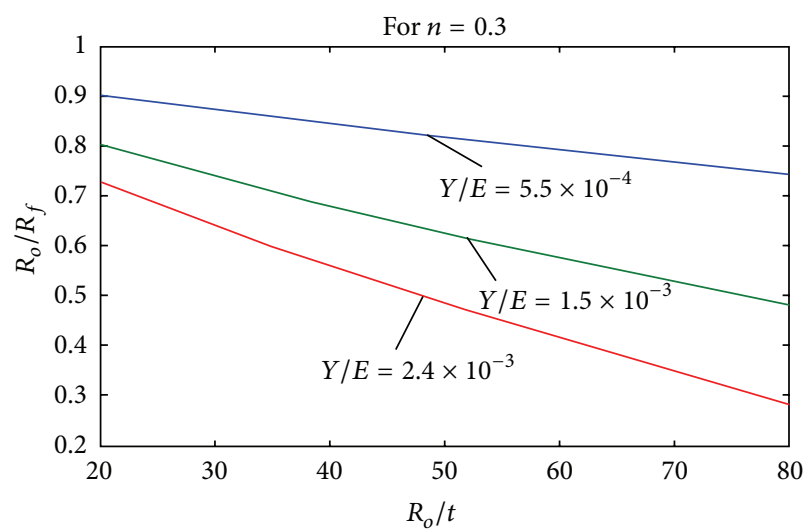

FIGURE 6: Springback ratio with $R_{o} / t$ with different values of $\sigma_{o} / E$ or $Y / E$ at $n=0.2, v=0.33$.

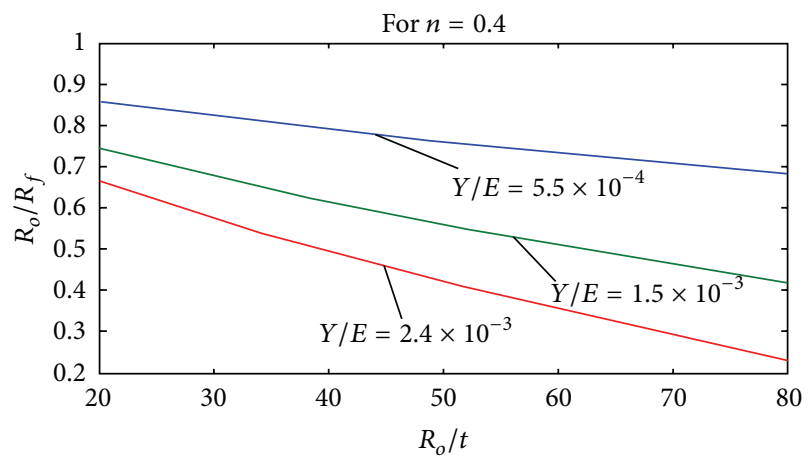

FIGURE 7: Springback ratio with $R_{o} / t$ with different values of $\sigma_{o} / E$ or $Y / E$ at $n=0.4, v=0.33$.

$$
\begin{gathered}
\times\left[\frac{3\left(1-v^{2}\right)^{n+3}}{(3 / 4)^{(n+1) / 2}(n+2)\left(1-v+v^{2}\right)^{(n+2) / 2}}\right. \\
\left.-\frac{\left(1-v^{2}\right)^{3}}{\left(1-v+v^{2}\right)^{3 / 2}}\right] .
\end{gathered}
$$

Here, from (8),

$$
K=\left[E\left(\frac{E}{\sigma_{o}}\right)^{n-1}\right] .
$$

Then, $K / E=\left(\sigma_{o} / E\right)^{1-n}$.

We can see that both equations have a last term that is very negligible because the $\sigma_{o} / E$ or $Y / E=5.5 \times 10^{-4}$ and its three times will be $10^{-12}$; therefore, we have to neglect the last term of both equations.

Then we write,

$$
\begin{aligned}
& \frac{R_{o}}{R_{f}}=1-\frac{3\left(1-v^{2}\right)}{(3 / 4)^{(1+n) / 2}(n+2)}\left(\frac{E}{Y}\right)^{n-1}\left(\frac{2 R_{o}}{t}\right)^{1-n}, \\
& \frac{R_{o}}{R_{f}}=1-\frac{3\left(1-v^{2}\right)}{(n+2)(3 / 4)^{(n+1) / 2}}\left(\frac{Y}{E}\right)^{1-n}\left(\frac{2 R_{o}}{t}\right)^{1-n} .
\end{aligned}
$$




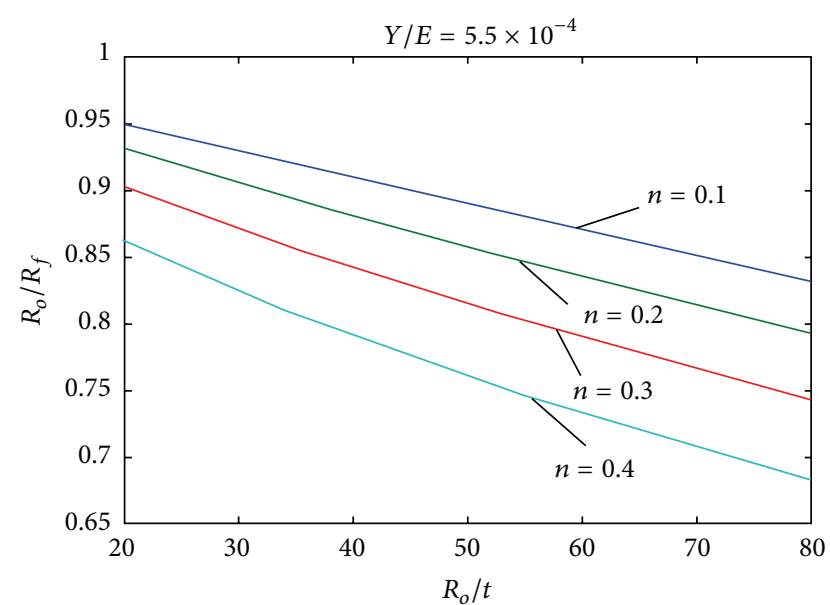

FIGURE 8: Springback ratio with $R_{o} / t$ with different values of $n$ at $Y / E=5.5 \times 10^{-4}, v=0.33$.

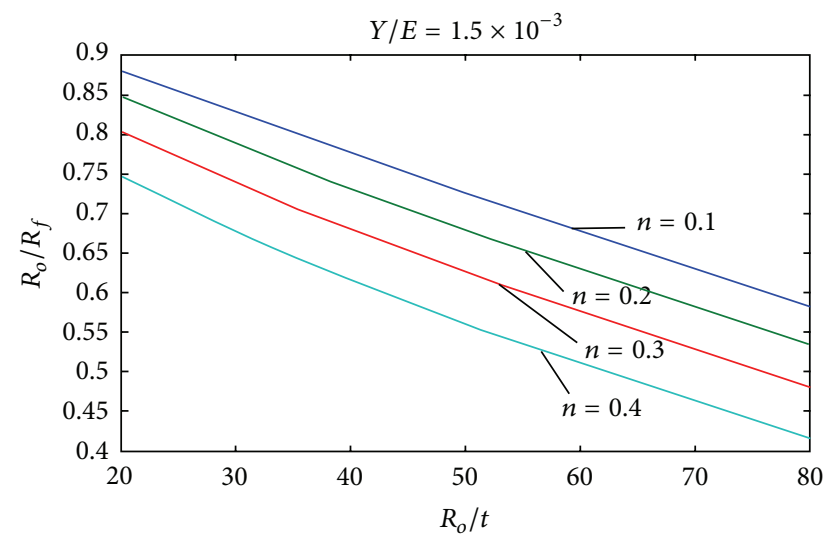

FIGURE 9: Springback ratio with $R_{o} / t$ with different values of $n$ at $Y / E=1.5 \times 10^{-3}, v=0.33$.

Here, we can see that both (62) and (63) are the same if we can apply Tresca or von Mises, getting same result but last term is different. The values of last term are much less; therefore, we neglect this term.

Therefore, we can say that if we apply Tresca or von Mises yield criteria, then we are getting same result. This equation is dependent on the $R_{o} / t, \sigma_{o} / E$, strain hardening coefficient $n$, and $\nu$ is Possion's ratio.

Figure $4(n=0.1)$, Figure $5(n=0.2)$, Figure $6(n=0.3)$, and Figure 7 ( $n=0.4$ ) show the variation in springback ratio along with change in $R_{o} / t$ with different $\sigma_{o} / E$ ratio. The higher value of $n$ (strain hardening coefficient) material to closer to elasto-ideal plastic material, and thickness is one $\mathrm{mm}$, and $\sigma_{o} / E$ value is $5.5 \times 10^{-4}, 1.522 \times 10^{-3}$, and $2.4 \times 10^{-3}$ (for different material c10100 copper, 1100al, and 1065steel). From the figure, we come to know that as the with increasing ratio of $R_{o} / t$ is decrease the springback ratio and with decreasing $\sigma_{o} / E$ springback ratio is increases.

Figures 8,9 , and 10 show the variation in springback ratio along with $R_{o} / t$ with the different value of $n$ with fixed value

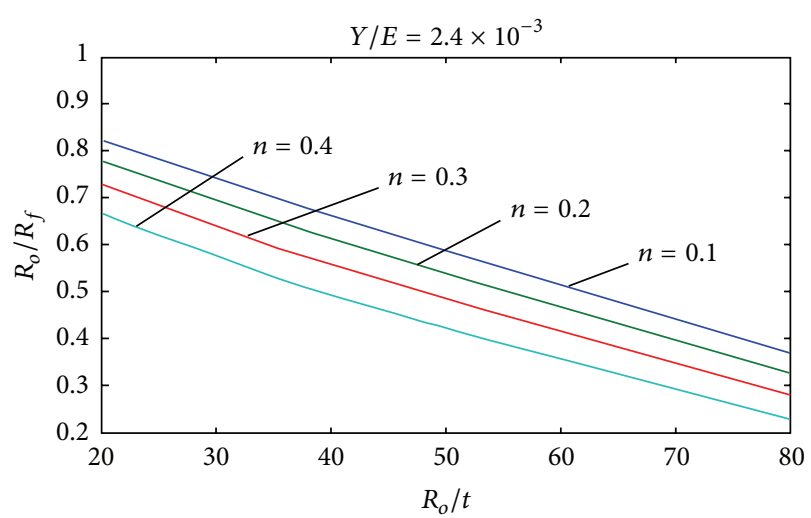

FIGURE 10: Springback ratio with $R_{o} / t$ with different values of $n$ at $Y / E=2.4 \times 10^{-3}, v=0.33$.

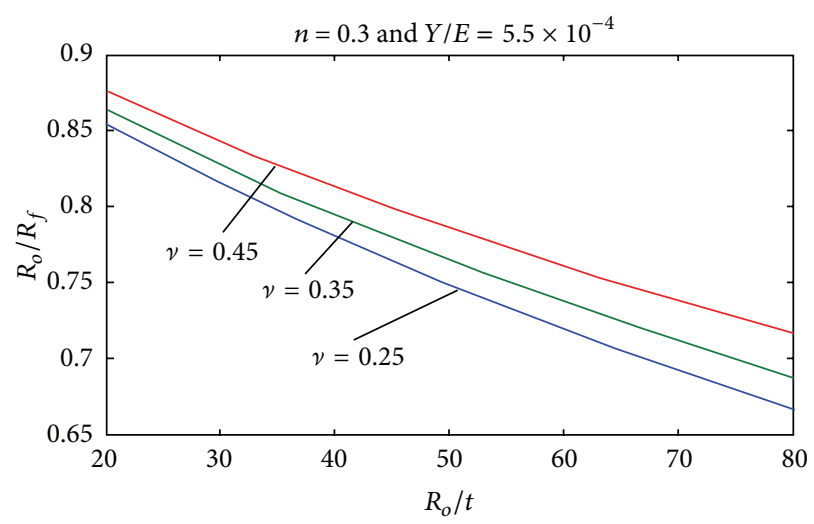

FIGURE 11: Springback ratio with $R_{o} / t$ with different values of $v$ at $Y / E=5.5 \times 10^{-4}$.

of $\sigma_{o} / E=5.5 \times 10^{-4}, \sigma_{o} / E=1.522 \times 10^{-3}$, and $\sigma_{o} / E=2.4 \times$ $10^{-3}$, here we take the values of $n=0.1,0.2,0.3$, and 0.4 . From the figure, we come to know that with increasing value of $n$, springback ratio is decreasing.

Figures 11, 12, and 13 show the variation in springback along with $R_{o} / t$ with different value of Possion's ratio $v=0.25$, $v=0.35$, and $v=0.45$ with fixed $\sigma_{o} / E=5.5 \times 10^{-4}$, $\sigma_{o} / E=1.522 \times 10^{-3}, \sigma_{o} / E=2.4 \times 10^{-3}$, and $n=0.3$. From the figure, we come to know that with increasing value of $v$, springback ratio is increasing.

Figures 14, 15, and 16 show the variation in springback along with $t$ with different value of $n=0.1,0.2,0.3$, and 0.4 and where $\sigma_{o} / E=5.5 \times 10^{-4}, \sigma_{o} / E=1.522 \times 10^{-3}$, $\sigma_{o} / E=2.4 \times 10^{-3}, \nu=0.33$, and $R_{o}=40$ is fixed. From the figure, we come to know that the springback ratio rapidly increases in range of 0 to $2 \mathrm{~mm}$ thickness, then the increase in springback ratio is stable.

\section{Conclusions}

Based on the results presented in chapter of results and discussion, following conclusions about the springback analysis in sheet metal forming using nonlinear constitutive equation, the following applies. 


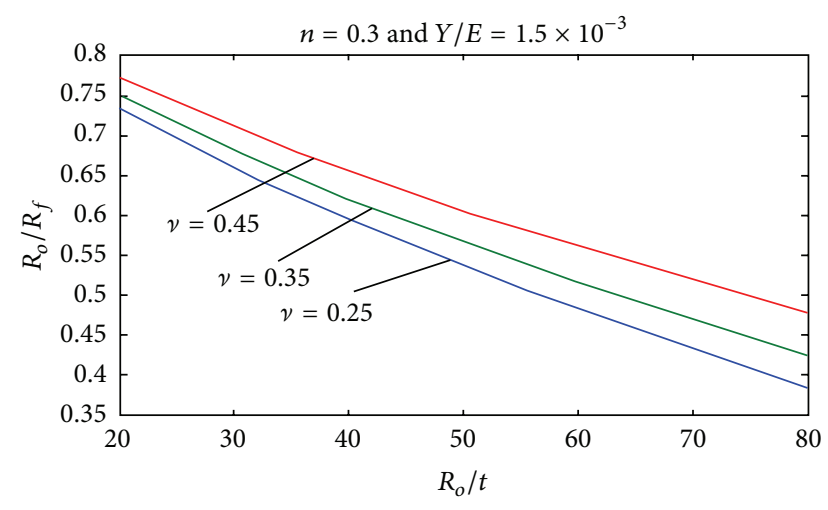

FIGURE 12: Springback ratio with $R_{o} / t$ with different values of $v$ at $Y / E=1.5 \times 10^{-3}$.

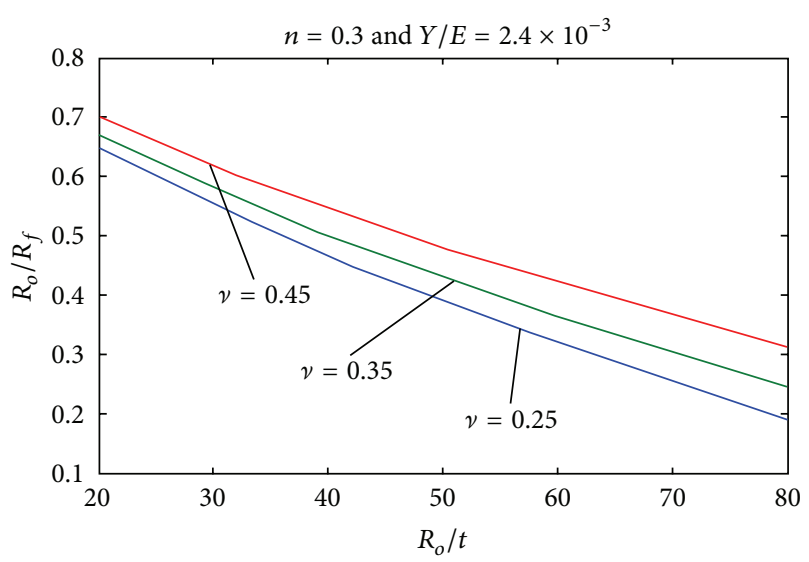

FIGURE 13: Springback ratio with $R_{o} / t$ with different values of $v$ at $Y / E=2.4 \times 10^{-3}$.

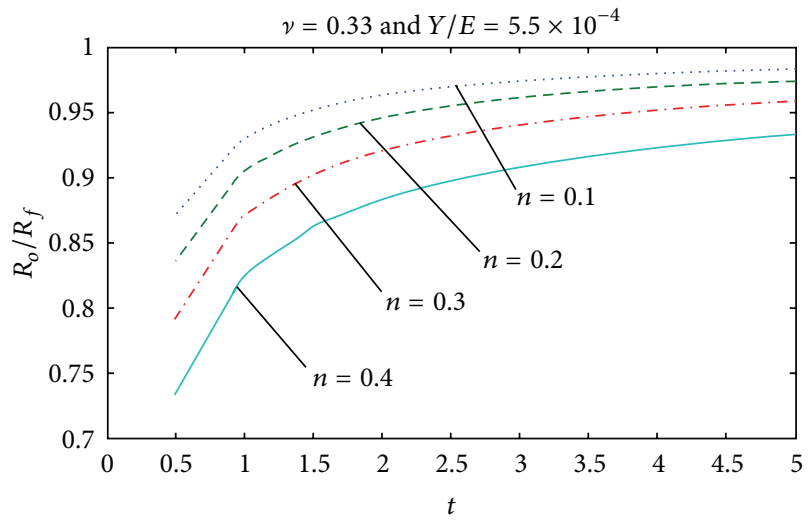

FIGURE 14: Springback ratio with $t$ for different values of $n$ at $Y / E=$ $5.5 \times 10^{-4}$.

(i) The theoretical analysis for the sheet metal under pure bending has been done, and it was found that the prediction of springback is quite successfull.

(ii) As expected, elastic recovery is found to be more with decreasing values of work hardening coefficient. At

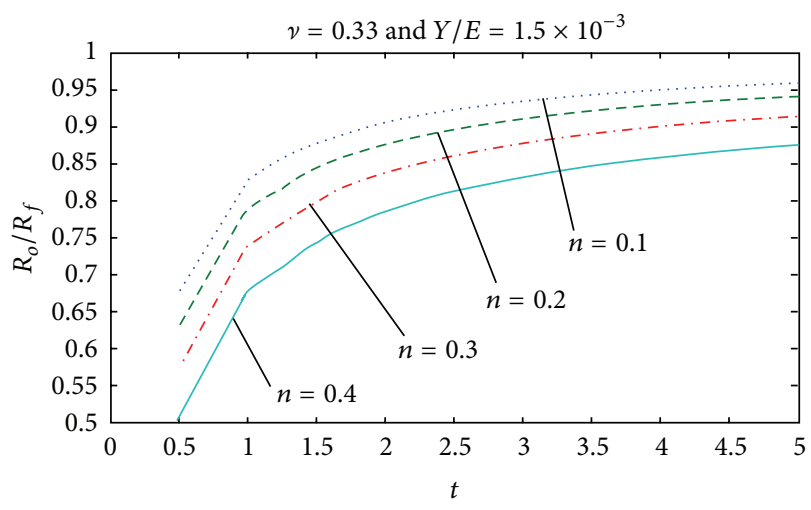

FIGURE 15: Springback ratio with $t$ for different values of $n$ at $Y / E=$ $1.5 \times 10^{-3}$.

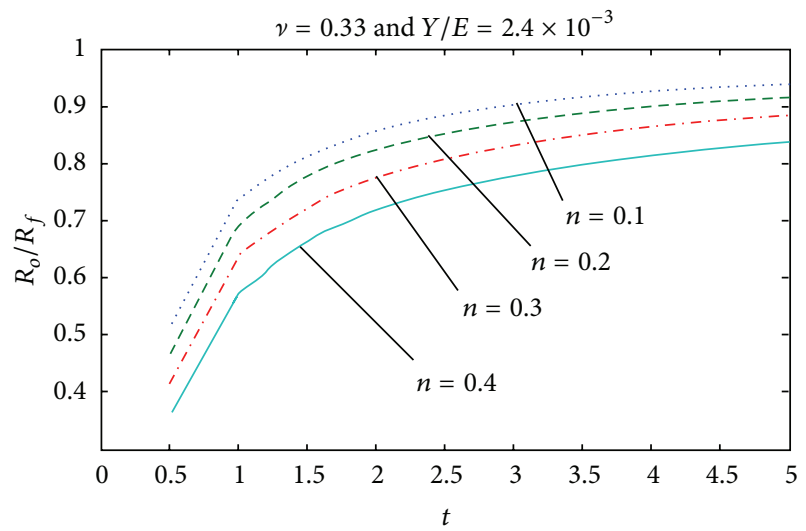

FIGURE 16: Springback ratio with $t$ for different values of $n$ at $Y / E=$ $2.4 \times 10^{-3}$.

lower values of $\mathrm{n}$, the material will approach to an elasto-ideally plastic behavior.

(iii) Springback ratio increases with increasing thickness.

(iv) Springback ratio increases with decreasing ratio of yield point stress to Young's modulus of elasticity

(v) Springback ratio is increasing with increasing Possion's ratio.

(vi) Springback ratio for Tresca and von Mises yield criteria, we say that $R_{o} / t$ is less than 20 , there will be slightly change in that is negligible and for high value of $R_{o} / t$ or more than 20 , differences in springback ratio is increases.

\section{Nomenclature}

$R_{o}$ : $\quad$ Radius of die

$R_{f}$ : Radius of final product after removing load

$M_{\text {max }}$ : Maximum applied bending moment per unit width

y: $\quad$ Distance from midsection

$t: \quad$ Thickness

$\sigma: \quad$ True stress

$\varepsilon: \quad$ True strain

E: Modulus of elasticity 


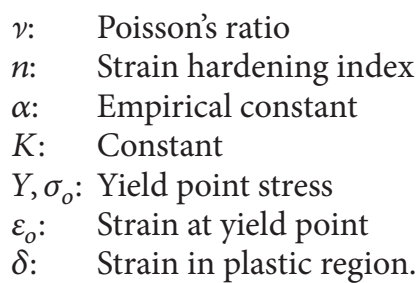

\section{References}

[1] G. Sachs, Principles and Methods of Sheet Metal Fabricating, Reinhold, New York, NY, USA, 1951.

[2] W. Schroeder, "Mechanics of sheet metal bending," Transactions of the ASME, vol. 36, pp. 138-145, 1943.

[3] F. J. Gardiner, "The springback of metals," Transactions of the ASEME, vol. 49, pp. 1-9, 1958.

[4] A. N. Singh and W. Johnson, "Springback after cylindrically bending metal strips," in Proceedings of the Dr Karunesh Memorial International Conference, New Delhi, India, December 1979.

[5] J. H. Huth, "A note on plastic torsion," Journal of Applied Mechanics, vol. 22, pp. 432-434, 1955.

[6] A. Nadai, Theory of Flow and Fracture of Solids, vol. 1, McGrawHill, 1950.

[7] P. C. Upadhyay, Elasto-plastic torsion [M.Tech. thesis], Mechanical Engineering Department, I.I.T. Kanpur, 1970.

[8] J. P. Dwivedi, A. N. Singh, S. Ram, and N. K. Das Talukder, "Springback analysis in torsion of rectangular strips," International Journal of Mechanical Sciences, vol. 28, no. 8, pp. 505-515, 1986.

[9] J. P. Dwivedi, P. K. Sarkar, R. S. Ram. S., N. K. D. Talukder, and A. N. Singh, "Experimental aspects of torsional springback in rectangular strips," Journal of the Institution of Engineers, vol. 67, no. 4, pp. 70-73, 1987.

[10] J. P. Dwivedi, A. K. Shukla, and P. C. Upadhyay, "Torsional springback of square section bars of linear wor hardening materials," Computers and Structures, vol. 45, no. 3, pp. 421-429, 1972.

[11] J. P. Dwivedi, P. C. Upadhyay, and N. K. Das Talukder, “Torsional springback in square section bars of nonlinear work-hardening materials," International Journal of Mechanical Sciences, vol. 32, no. 10, pp. 863-876, 1990.

[12] J. P. Dwivedi, P. C. Upadhyay, and N. K. D. Talukder, "Springback analysis of torsion of L-sectioned bars of work-hardening materials," Computers and Structures, vol. 43, no. 5, pp. 815-822, 1992.

[13] Z. T. Zhang and S. J. Hu, "Stress and residual stress distributions in plane strain bending," International Journal of Mechanical Sciences, vol. 40, no. 6, pp. 533-543, 1998.

[14] T. Kuwabara, "Advances in experiments on metal sheets and tubes in support of constitutive modeling and forming simulations," International Journal of Plasticity, vol. 23, no. 3, pp. 385419, 2007.

[15] H. K. Yi, D. W. Kim, C. J. van Tyne, and Y. H. Moon, "Analytical prediction of springback based on residual differential strain during sheet metal bending," Journal of Mechanical Engineering Science, vol. 222, no. 2, pp. 117-129, 2008.

[16] A. El Megharbel, G. A. El Nasser, and A. El Domiaty, "Bending of tube and section made of strain-hardening materials," Journal of Materials Processing Technology, vol. 203, no. 1-3, pp. 372-380, 2008.
[17] E. Da-xin, H. Hau-hui, L. Xiao-yi, and N. Ru-xin, "Spring-back deformation in tube bending," International Journal of Minerals, Metallurgy and Materials, vol. 16, no. 2, pp. 177-183, 2009.

[18] D. E. Daxin E and Y. Liu, "Springback and time-dependent springback of $1 \mathrm{Cr} 18 \mathrm{Ni} 9 \mathrm{Ti}$ stainless steel tubes under bending," Materials and Design, vol. 31, no. 3, pp. 1256-1261, 2010.

[19] V. K. Choubey, M. Gangwar, and J. P. Dwivedi, "Torsional springback analysis in thin tubes with non-linear work hardening," Journal of Mechanical Engineering, vol. 7, no. 1, pp. 15-34, 2011.

[20] V. K. Choubey, M. Gangwar, and J. P. Dwivedi, "Springback analysis of thin tubes," Journal of Mechanical Engineering, vol. 7, no. 2, 2011.

[21] V. K. Choubey, M. Gangwar, J. P. Dwivedi, and N. K. das Talukder, "Springback analysis of thin tubes with arbitrary stress-strain curves," Journal of Mechanical Engineering, vol. 8, no. 1,2011 

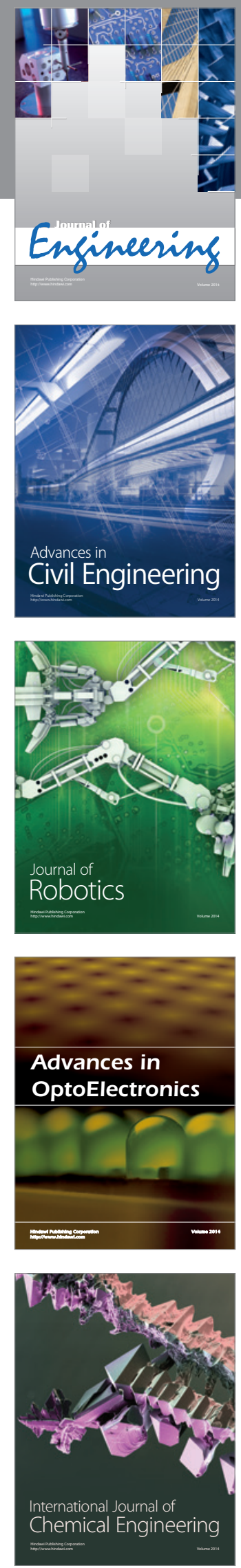

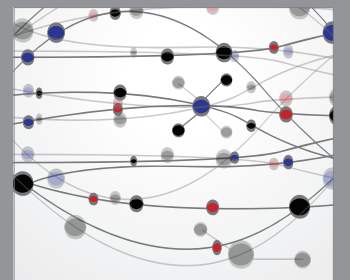

The Scientific World Journal
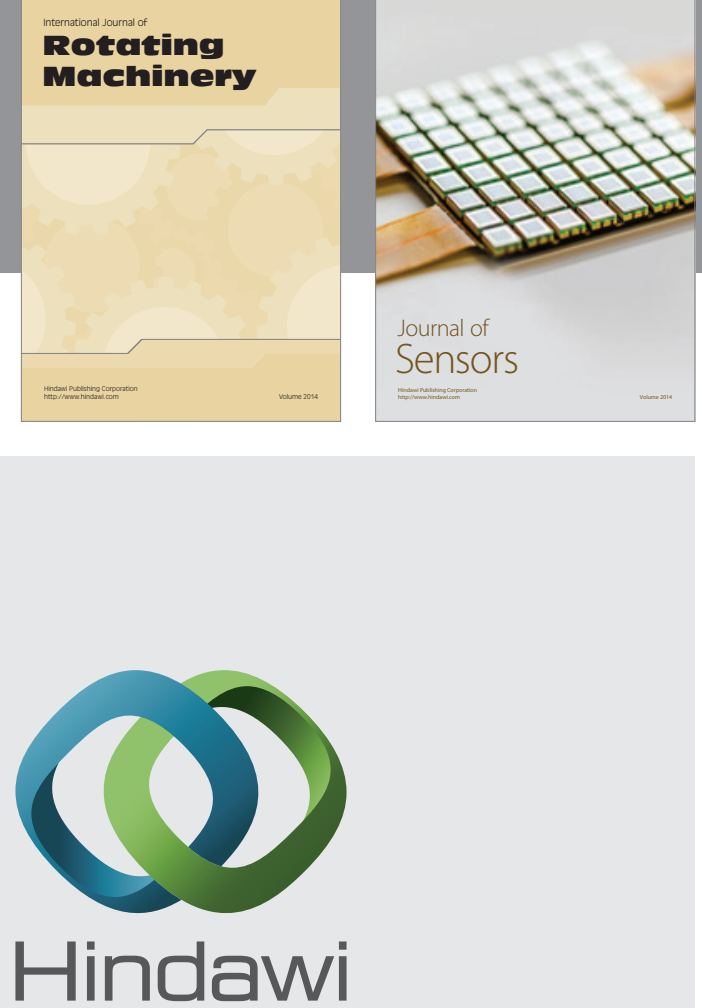

Submit your manuscripts at http://www.hindawi.com
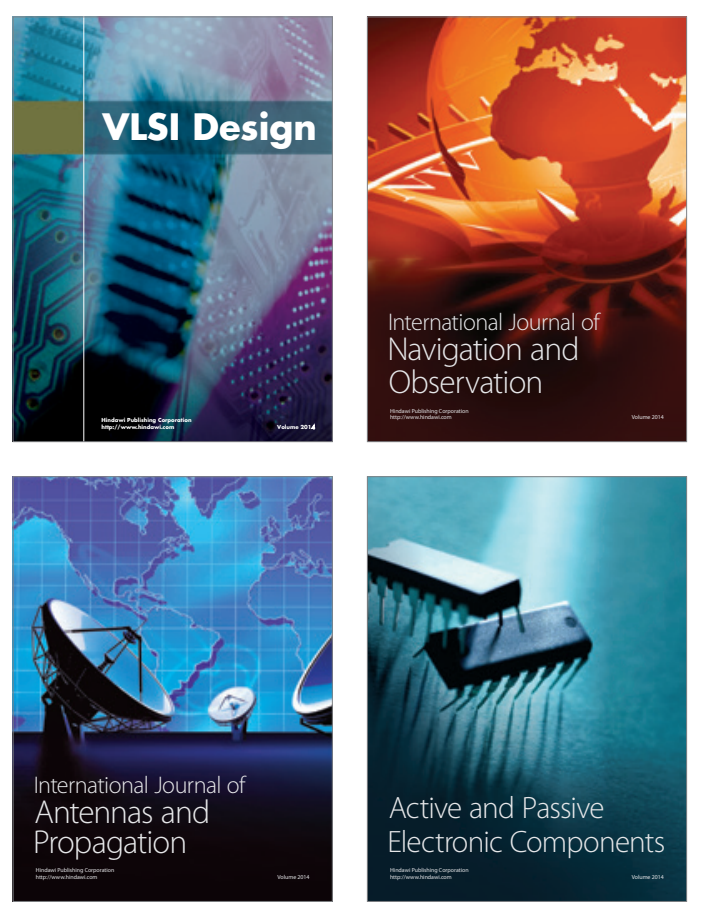
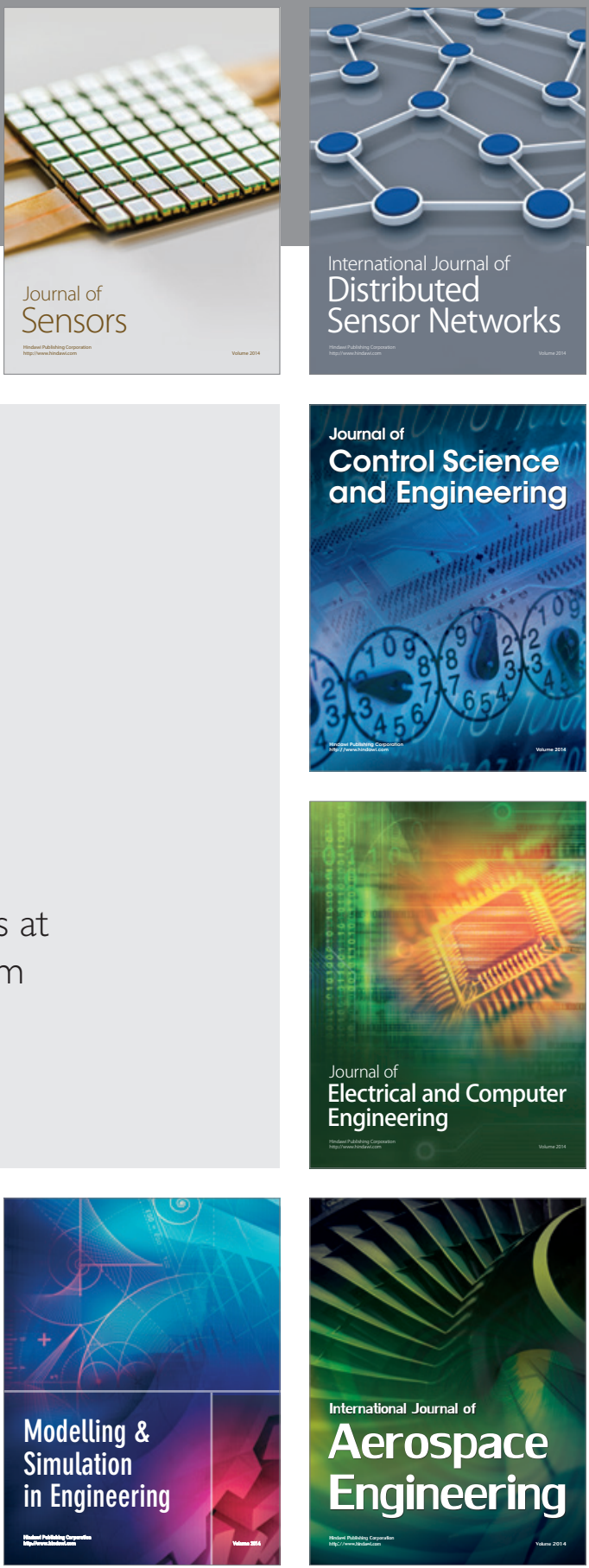

Journal of

Control Science

and Engineering
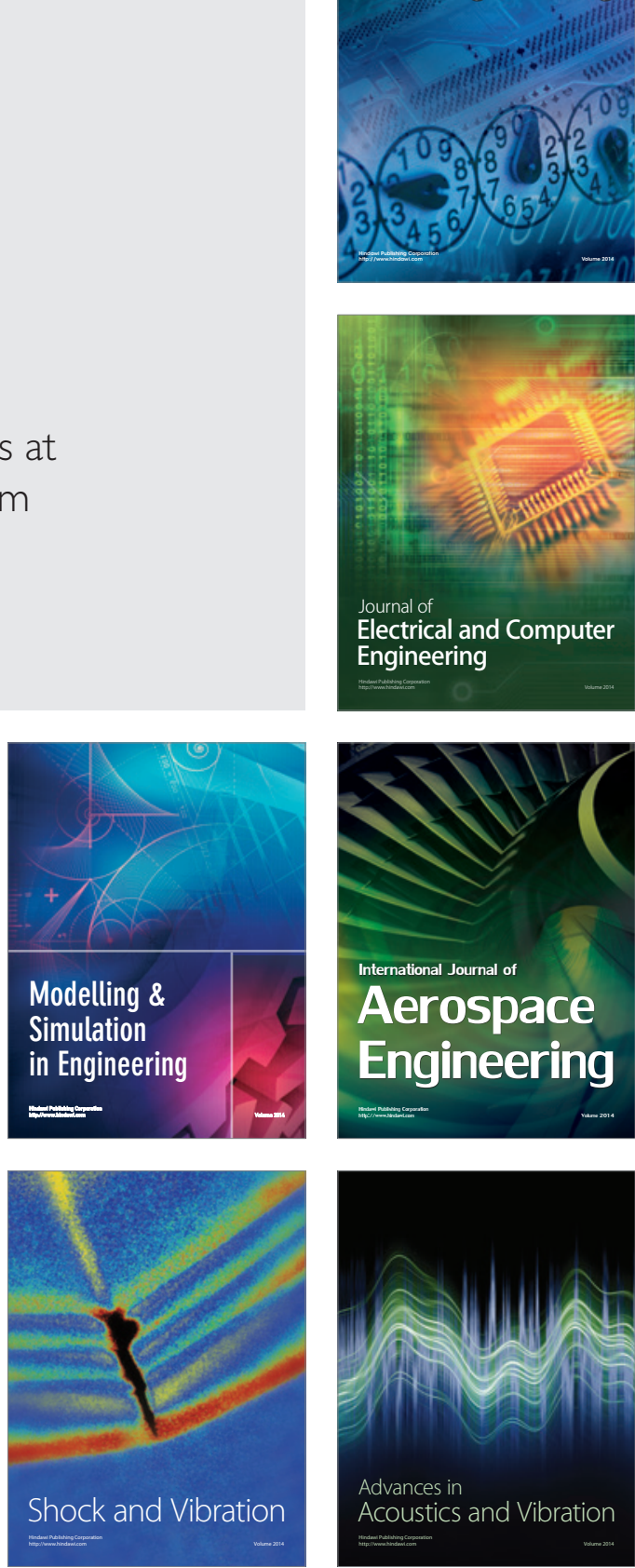